\title{
Closure Relations for the Eigenfunctions of the One-Speed Transport Equation
}

\author{
I. KUŠČER* AND F. SHURE \\ Department of Nuclear Engineering, The University of Michigan, Ann Arbor, Michigan
}

(Received 13 May 1966)

\begin{abstract}
Orthogonality relations for the eigenfunctions of the one-speed transport equation are used to derive the corresponding closure relations. These express in a concise form the completeness properties previously proved by Case.
\end{abstract}

\section{INTRODUCTION}

$\mathrm{T}$ HE plane-symmetric normal mode solutions to the equation of steady-state one-speed neutron transport with isotropic scattering possess a variety of completeness properties. These were all proved by Case $^{1}$ via construction of a solution to the singular integral equation for the expansion coefficients implied by a hypothetical representation of an arbitrary function in terms of some set of the eigenfunctions. In such an approach the form of the expansion coefficients appears as a by-product of the completeness proof. In a later paper $^{2}$ (henceforth referred to as I), a set of orthogonality relations was presented, which may be used to determine expansion coefficients directly. On the other hand, as we show, these relations also provide a completeness proof in a more conventional form. That is, by the use of our knowledge of the normalization coefficients contained in the orthogonality relations, we exhibit the closure property for Case's eigenfunctions by direct calculation. Acquaintance with paper $I$ is assumed, and definitions and notation are borrowed from there.

First, in Sec. II we show that the set of continuum eigenfunctions $\left\{\phi_{v}(\mu)\right\}, \alpha \leq v \leq \beta$, obey a closure relation with respect to the interval $[\alpha, \beta]$ whenever $\alpha$ and $\beta$ both lie in the open interval $(-1,1)$. Then, in Sec. III we discuss the lack of uniqueness or "overcompleteness" that arises when $\alpha$ and $\beta$ are both positive or both negative, and indicate the modifications necessary when $\alpha$ or $\beta$ take on the special values \pm 1 .

\section{PARTIAL RANGe COMPLETENESS}

The expansion coefficient $A(v)$ in

$$
\psi(\mu)=\int_{\alpha}^{\beta} A(\nu) \phi_{v}(\mu) d \nu
$$

is conveniently calculated by application of the

\footnotetext{
* Permanent address: Institute of Physics, University of Ljubljana, Ljubljana, Yugoslavia.

${ }^{1}$ K. M. Case, Ann. Phys. (N.Y.) 9, 1 (1960)

2 I. Kuščer, N. J. McCormick, and G. C. Summerfield, Ann. Phys. (N.Y.) 30, 411 (1964).
}

orthogonality relation [Eq. (I.22)]

$$
\int_{\alpha}^{\beta} \phi_{v}(\mu) \phi_{v^{\prime}}(\mu) \gamma_{0}(\mu) d \mu=\gamma_{0}(v) \Lambda^{+}(v) \Lambda^{-}(v) \delta\left(v-v^{\prime}\right) .
$$

(Here and henceforth we choose for the principalvalue integral with two singularities in the integrand the same interpretation as used by Case. ${ }^{1.3}$ ) From the right-hand side of Eq. (1) we obtain

$$
\begin{aligned}
\int_{\alpha}^{\beta} \phi_{v^{\prime}}(\mu) \gamma_{0}(\mu) d \mu \int_{\alpha}^{\beta} A(\nu) & \phi_{v}(\mu) d v \\
& =A\left(v^{\prime}\right) \gamma_{0}\left(\nu^{\prime}\right) \Lambda^{+}\left(v^{\prime}\right) \Lambda^{-}\left(v^{\prime}\right) .
\end{aligned}
$$

The procedure is legitimate whenever the functions involved are such that the order of integration on the left-hand side of the last equation can be reversed by application of the Poincaré-Bertrand formula. ${ }^{4}$

If the above method is formally applied to the function $\psi(\mu)=\delta\left(\mu-\mu^{\prime}\right)$, a closure relation immediately follows:

$$
\begin{aligned}
\int_{\alpha}^{\beta} \phi_{v}(\mu) \phi_{v}\left(\mu^{\prime}\right) & \frac{\gamma_{0}\left(\mu^{\prime}\right)}{\gamma_{0}(v)} \frac{d v}{\Lambda^{+}(v) \Lambda^{-}(v)}=\delta\left(\mu-\mu^{\prime}\right), \\
& \mu, \mu^{\prime} \in[\alpha, \beta], \quad-1<\alpha<\beta<1 .
\end{aligned}
$$

Our main goal is to prove this identity in a direct way. We may remark that the convergence of the integral for $\mu \neq \mu^{\prime}$ is ensured since the factor $\nu\left[\gamma_{0}(\nu)\right]^{-1}$ possesses at most a weak (i.e., integrable) singularity (cf., the discussion at the beginning of Sec. III), whereas the factor $\left[\Lambda^{+} \Lambda^{-}\right]^{-1}$ is well behaved.

Substituting the expression (I.2c) [i.e., Eq. (2c) of Ref. I. This notation is applied several times throughout this paper] for $\phi_{v}(\mu)$, we obtain two $\delta$-function contributions from the left-hand side of Eq. (4): a term

$$
\left[\lambda^{2}(\mu) / \Lambda^{+}(\mu) \Lambda^{-}(\mu)\right] \delta\left(\mu-\mu^{\prime}\right)
$$

due to the product of the $\delta$ functions appearing explicitly in $\phi_{v}$, and a term

$$
\left[\pi c^{2} \mu^{2} / 4 \Lambda^{+}(\mu) \Lambda^{-}(\mu)\right] \delta\left(\mu-\mu^{\prime}\right)
$$

\footnotetext{
${ }^{3}$ I. Kušcer and N. J. McCormick, Nucl. Sci. Eng. 23, 404 (1965).

4 N. I. Muskhelishvili, Singular Integral Equations (P. Noordhoff, Ltd., Groningen, The Netherlands, 1953).
} 
arising from the application of Eq. (I.9). According to Eq. (I.4b), both contributions together give just the right-hand side of (4).

We are left with the task of showing that the remaining part of the integral in (4) vanishes. Since this part has the form

$$
\begin{aligned}
I(\mu)=\frac{c \mu}{2} & \frac{\lambda(\mu)}{\gamma_{0}(\mu) \Lambda^{+}(\mu) \Lambda^{-}(\mu)} \\
& \quad+P \int_{\alpha}^{\beta}\left(\frac{c v}{2}\right)^{2} \frac{1}{\gamma_{0}(v) \Lambda^{+}(v) \Lambda^{-}(v)} \frac{d v}{v-\mu},
\end{aligned}
$$$$
\text { where }
$$$$
\gamma_{0}\left(\mu^{\prime}\right)\left[I(\mu)-I\left(\mu^{\prime}\right)\right] /\left(\mu-\mu^{\prime}\right)
$$

we only need to demonstrate that $I(\mu)$ is a constant.

In view of the identities

$$
\begin{aligned}
\frac{1}{X_{0}^{+}(v)} \pm \frac{1}{X_{0}^{-}(v)} & =\frac{c v}{2} \frac{\Lambda^{-}(v) \pm \Lambda^{+}(v)}{\gamma_{0}(v) \Lambda^{+}(v) \Lambda^{-}(v)} \\
& = \begin{cases}\frac{c v \lambda(v)}{\gamma_{0}(v) \Lambda^{+}(v) \Lambda^{-}(v)}, & (7+) \\
-2 \pi i\left(\frac{c v}{2}\right)^{2} \frac{1}{\gamma_{0}(v) \Lambda^{+}(v) \Lambda^{-}(v)}, & (7-)\end{cases}
\end{aligned}
$$

which are obtained from Eqs. (I.4b) and (I.17), the integral term on the right-hand side of (6) is equal to

where

$$
-\frac{1}{2}\left[N_{0}^{+}(\mu)+N_{0}^{-}(\mu)\right]
$$

$$
N_{0}(z) \equiv \frac{1}{2 \pi i} \int_{\alpha}^{\beta}\left[\frac{1}{X_{0}^{+}(v)}-\frac{1}{X_{0}^{-}(v)}\right] \frac{d v}{v-z} .
$$

This may be written also as

$$
N_{0}(z)=\frac{1}{2 \pi i} \int_{C} \frac{1}{X_{0}\left(z^{\prime}\right)} \frac{d z^{\prime}}{z^{\prime}-z},
$$

where the integration is carried out in the clockwise direction over a closed loop which surrounds the cut $(\alpha, \beta)$ but leaves the point $z$ outside. We deform this contour into a large circle to obtain

$$
N_{0}(z)=\left[1 / X_{0}(z)\right]-1,
$$

where we have used the fact that $X_{0}(z)$ possesses no zeros in the cut plane and tends to 1 as $z \rightarrow \infty$. Therefore,

$$
\frac{1}{2}\left[N_{0}^{+}(\mu)+N_{0}^{-}(\mu)\right]=\frac{1}{2}\left[\frac{1}{X_{0}^{+}(\mu)}+\frac{1}{X_{0}^{-}(\mu)}\right]-1,
$$

and by the use of identity $(7+)$ we see that the variable term here just cancels the other term in (6). Thus

$$
I(\mu)=1
$$

remains, so that the expression (5) vanishes, and the closure relation (4) is verified.

\section{OVERCOMPLETENESS AND UNDERCOMPLETENESS}

We still have to see under what conditions the closure relation (4) is unique, and what happens when $\alpha=-1$ or $\beta=1$, or both. To this end we reexamine the proof of the preceding section to ascertain when it may be, or may need to be, modified.

Since closure implies the mutual orthogonality of all the eigenfunctions involved, the question of uniqueness is answered simply by trying out all weight functions which produce orthogonality. These functions are listed in paper $I$; and if they are derived by the constructive method of Case and Zweifel, ${ }^{5}$ it can be seen that the list is complete.

The analysis hinges on the behavior of the function $\gamma_{0}(\nu)$ on the interval $[\alpha, \beta]$ over which it is defined. It can be seen ${ }^{1}$ that $\gamma_{0}(v)$ is real finite, and nonvanishing in the interior of the interval, except for the trivial zero at $v=0$. At the end points this function has, at most, weak zeros or weak infinities. That is to say,

$$
\begin{aligned}
\gamma_{0}(\nu) & \sim(\beta-\nu)^{\gamma(\beta)}, \quad \nu \rightarrow \beta, \\
& \sim(\nu-\alpha)^{-f(\alpha)}, \quad \nu \rightarrow \alpha .
\end{aligned}
$$

As long as $\alpha>-1$ and $\beta<1$, the exponents here are $<1$ in magnitude. In fact $\tau(x)$ is an odd monotone function with $\tau(1)=1$.

In view of the relation

$$
\phi_{v}(-\mu)=\phi_{-v}(\mu),
$$

no generality is lost by restricting ourselves to the case $\beta>0$. Let us try, still for $-1<\alpha<\beta<1$, to repeat the derivation of Eq. (4), with $X_{0}(z)$ substituted by an $a$ arbitrary $X$ function obeying the conditions mentioned in I:

$$
\begin{array}{ll}
X(z)=\frac{(a-b z)(c-d z)}{(z-\alpha)(\beta-z)} X_{0}(z), & \text { if } \quad \alpha<0, \\
X(z)=\frac{(a-b z)}{(\beta-z)} X_{0}(z), & \text { if } \quad \alpha \geq 0,
\end{array}
$$

with arbitrary $a, b, c, d$. The corresponding functions $\gamma(y)$ and $N(z)$ are defined by Eq. (I.15a) and by an equation of the form (9), respectively. In the analog of Eq. (11), an additional term containing $z$ crops up. due to the residues of the integrand at $z=a / b$ and $z=c / d$ (at infinity if $b=0$ or $d=0$ ). Consequently the term corresponding to (5) now does not vanish.

We have tacitly avoided the choice $a / b=c / d=\alpha$ or $\beta$ in Eq. (16a) or $a / b=\alpha$ in (16b). In view of Eq. (14), whenever $\alpha \leq 0$, such a choice causes the

${ }^{5}$ K. M. Case and P. F. Zweifel, Linear Transport Theory (AddisonWesley Publishing Company, Inc., Reading, Massachusetts, 1967). 
function $\gamma(v)$ to acquire a strong zero at the respective end point, which prevents the integral in Eq. (4) from converging. Thus, although orthogonality is produced by three linearly independent weight functions if $\alpha<0$, or by two if $\alpha=0$, in these two cases $\gamma_{0}(v)$ is the only such function allowed in the closure relation (4). This agrees with Case's statement ${ }^{1}$ that for any such interval the expansion (1) is unique.

An apparent paradox arises when orthogonality relations with different weight functions are applied to Eq. (1). In contradiction to the established uniqueness of this expansion, different expansion coefficients $A(v)$ seem to follow. However, a closer inspection shows that, although orthogonality relations are formally valid for any of those weight functions $\gamma(\mu)$, only one of them, $\gamma_{0}(\mu)$, can be used for the determination of expansion coefficients. The trouble stems from the weak infinities of $A(v)$ and of the "useless" weight functions at one of the end points, where, e.g., a behavior like $(\beta-\nu)^{-\tau(\beta)}$ and $(\beta-\mu)^{-1+\tau(\beta)}$ is encountered. The two exponents add up to -1 , whereby the Poincaré-Bertrand formula breaks down, ${ }^{4}$ and consequently the equation corresponding to (3) becomes invalid. (The integral over $\mu$ on the left-hand side diverges.)

The situation is different if $\alpha>0$, because then the function

$$
\gamma_{1}(\nu) \equiv[(\nu-\alpha) /(\beta-v)] \gamma_{0}(v)
$$

is a valid substitute for $\gamma_{0}(v)$, and we indeed get two closure relations. Similarly, for an arbitrary $\psi(\mu)$ we obtain two different expansions (1), one from the orthogonality relation with the weight $\gamma_{0}(\mu)$, and the other with $\gamma_{1}(\mu)$. Their difference gives an expansion with the sum zero,

$$
\begin{gathered}
\int_{\alpha}^{\beta} A(v) \phi_{v}(\mu) d v=0, \\
A(v) \propto \frac{c v}{2} \frac{1}{(v-\alpha) \gamma_{0}(v) \Lambda^{+}(v) \Lambda^{-}(v)},
\end{gathered}
$$

as can be verified directly. We may say that the set $\left\{\phi_{v}(\mu)\right\}, \alpha \leq \nu \leq \beta$, now is overcomplete (not linearly independent) in the interval $\alpha \leq \mu \leq \beta .^{1}$

It should be emphasized that for $0<\alpha<\beta<1$ only the two weight functions $\gamma_{0}(\mu)$ and $\gamma_{1}(\mu)$ are useful for determining the expansions of $\psi(\mu)$. Linear combinations of $\gamma_{0}$ and $\gamma_{1}$, although formally permitted in the orthogonality relation (2), fail in Eq. (3), because again the Poincaré-Bertrand formula breaks down at one of the end points. For the same reason, none of the orthogonality relations can be applied to linear combinations of the two expansions. In particular, this warning holds for Eqs. (18), (19), which therefore do not contradict orthogonality.
Whenever $\beta=1$, the proof given in Sec. II breaks down in its first step, since $\gamma_{0}(v) \sim(1-\nu)$ in the neighborhood of $v=1$ and the integral in (4) diverges. However, in the particular case $0<\alpha<\beta=1$ the closure relation (4) is valid with $\gamma_{0}(v)$ replaced by $\gamma_{1}(v)$, Eq. (17). Of course, the proof is the same as before. Hence, here again we have unique expansions in terms of the continuum modes alone, in agreement with Case.

We consider finally the case $\beta=1, \alpha \leq 0$, and try to use again a general $X$ function and a corresponding $\gamma$ function. It turns out that no such combination leads to a closure relation of the form (4), because either the integral there diverges, or a residue of the reciprocal $X$ function leads to an additional term. We are thus forced to the conclusion that the continuum eigenfunctions alone no longer form a complete set. ${ }^{1}$

However, the set $\left\{\phi_{v}\right\}$ may be made complete by the addition of only one (two, in the case $\alpha=-1$, $\beta=1$ ) new function, linearly independent of the continuum $\left\{\phi_{v}\right\}$. One such function is the discrete eigenfunction

$$
\phi_{+}(\mu)=\frac{1}{2} c \gamma_{0}\left[1 /\left(\nu_{0}-\mu\right)\right],
$$

which is automatically introduced by repeating the derivation of Sec. II with

$$
X_{2}(z) \equiv\left[\left(v_{0}-z\right) /(1-z)\right] X_{0}(z),
$$

and with the corresponding functions $\gamma_{2}(\mu)$ and $N_{2}(z)$. (For simplicity we exclude the case $c=1$, so that $v_{0} \neq \infty$.) When proceeding from Eq. (10) to Eq. (11) we get an extra term, namely the residue of the integrand at $z^{\prime}=v_{0}$. Let us quote the final result for the halfrange $(\alpha=0, \beta=1)$ :

$$
\begin{aligned}
& \gamma_{2}\left(\mu^{\prime}\right)\left[\int_{0}^{1} \phi_{v}(\mu) \phi_{v}\left(\mu^{\prime}\right) \frac{d v}{\gamma_{2}(\nu) \Lambda^{+}(v) \Lambda^{-}(v)}\right. \\
& \left.\quad+\phi_{+}(\mu) \phi_{+}\left(\mu^{\prime}\right)\left(\frac{2}{c v_{0}}\right)^{2} \frac{v_{0}-1}{X_{0}\left(v_{0}\right)}\right]=\delta\left(\mu-\mu^{\prime}\right) .
\end{aligned}
$$

This agrees with what follows from the orthogonality relations (I.A1), (I.A2), (I.A4), if the different notation used there is taken into account:

$$
\gamma(z)=\gamma_{0}(z) /(1-z)=\gamma_{2}(z) /\left(v_{0}-z\right) .
$$

In the full-range case $(\alpha=-1, \beta=1)$, two discrete terms are needed for completion, for instance the two discrete eigenfunctions $\phi_{+}(\mu)$ and $\phi_{-}(\mu)$. These are introduced by taking

$$
X_{3}(z) \equiv \frac{v_{0}^{2}-z^{2}}{1-z^{2}} X_{0}(z)=\frac{\Lambda(z)}{1-\mathcal{c}} .
$$


Pursuing the same procedure as before we obtain

$$
\begin{array}{r}
\mu^{\prime}\left\{\int_{-1}^{1} \phi_{v}(\mu) \phi_{v}\left(\mu^{\prime}\right) \frac{d v}{v \Lambda^{+}(v) \Lambda^{-}(v)}\right. \\
\left.+\left[\phi_{+}(\mu) \phi_{+}\left(\mu^{\prime}\right)-\phi_{-}(\mu) \phi_{-}\left(\mu^{\prime}\right)\right] \frac{2}{c v_{0}^{2} \Lambda^{\prime}\left(v_{0}\right)}\right\} \\
=\delta\left(\mu-\mu^{\prime}\right),
\end{array}
$$

where

$$
\Lambda^{\prime}\left(v_{0}\right) \equiv\left[\frac{d \Lambda(z)}{d z}\right]_{z=v_{0}}=\frac{c \nu_{0}}{v_{0}^{2}-1}-\frac{1}{v_{0}} .
$$

The result is seen to correspond to the known orthogonality relations for the full range case. ${ }^{1}$

Recollecting what has been said about completeness of the eigenfunctions we recover the classification given by Case. In short, we can state that the set of continuum modes $\phi_{v}(\mu), \alpha \leq v \leq \beta,-1 \leq \alpha<\beta \leq 1$, with respect to expansions in the interval $\alpha \leq \mu \leq \beta$,

is overcomplete, whenever this interval does not contain any of the three special points $-1,0,1$;

is complete if the interval contains one of those points;

is undercomplete if it contains two (or all three) of those points, and can be completed by addition of one (or two) further function, e.g., $\phi_{+}(\mu)$ or $\phi_{-}(\mu)$ (or both).

\section{COMMENTS}

The discussed closure relations are not entirely new, certainly not for the full range and the half range [Eqs. (24) and (22)]. For these two cases they are encountered in connection with the Green's function for the infinite medium and with the solution of the albedo problem for the semi-infinite medium, respectively. ${ }^{1.5}$ Hence, the above considerations represent nothing more than a demonstration that the $\delta$ function is truly reproduced by the expansions. So is any other function which we would expand.

Instead of deriving the closure relation from the orthogonality relations, one could alternatively prove the closure relation first, and derive the orthogonality relations therefrom. The factor needed in the integrand of Eq. (4) can be constructed in a way very close to that used by Case and $\mathrm{Zweife}^{5}$ for the orthogonality relations. Such an approach may have some pedagogic value as a short way of introducing Case's formalism from the start. A brief sketch is given in the following.

The eigenfunctions $\phi_{v}(\mu)$ satisfy the equation

$$
(\nu-\mu) \phi_{v}(\mu)=\frac{1}{2} c \nu,
$$

which we write down twice, for the values $\mu$ and $\mu^{\prime}$. We multiply both sides of the first equation by

$$
(2 / c v) \phi_{v}\left(\mu^{\prime}\right) G(v) d v
$$

and of the second by

$$
(2 / c v) \phi_{v}(\mu) G(v) d v
$$

where $G(v)$ is a function to be determined later. After integration and subtraction we obtain

$$
\begin{aligned}
\left(\mu-\mu^{\prime}\right) \int_{\alpha}^{\beta} & \frac{2}{c \nu} \phi_{v}(\mu) \phi_{v}\left(\mu^{\prime}\right) G(v) d v \\
& =\int_{\alpha}^{\beta} \phi_{v}(\mu) G(\nu) d v-\int_{\alpha}^{\beta} \phi_{v}\left(\mu^{\prime}\right) G(\nu) d v .
\end{aligned}
$$

If a closure relation with the "weight" $(2 / c v) G(v)$ exists, the integral on the left-hand side is zero for $\mu \neq \mu^{\prime}$. Hence we must require that

$$
\int_{\alpha}^{\beta} \phi_{v}(\mu) G(\nu) d \nu=\text { const. }
$$

This is a singular integral equation, like those considered by Case. By applying the usual technique ${ }^{1.5}$ we find that the solutions $G(v)$ indeed are the same as implied by the results of Secs. II and III.

The difference between Eq. (28) and the equation involved in the constructive determination of the orthogonality relations $s^{5}$ lies in the interchanged role of the variables $\nu$ and $\mu$, which means that the equations are the adjoints of each other, in Muskhelishvili's sense. ${ }^{4}$ This explains why the number of linearly independent orthogonality relations in general differs from the number of linearly independent closure relations.

Let us conclude with remarks about possible generalizations. A closure relation for the two-media case $^{2}$ can immediately be written down. Also the more general scheme with a variable $c(v)$, useful with a simple model of energy-dependent neutron transport or of nongrey radiative transfer, ${ }^{6,7}$ can be worked out without difficulty. In addition, a generalization to anisotropic scattering ${ }^{8,9}$ is also possible.

\section{ACKNOWLEDGMENTS}

We owe sincere thanks to Professor P. F. Zweifel and Professor G. C. Summerfield for enlightening discussion and encouragement, and for critical comment which enabled us to avoid some inconsistency.

One of us (I. K.) wishes to express appreciation of the department of Nuclear Engineering, University to Michigan, for the hospitality extended to him during his stay

\footnotetext{
${ }^{6}$ R. J. Bednarz and J. R. Mika, J. Math. Phys. 4, 1285 (1963); J. R. Mika, Nucl. Sci. Eng. 22, 235 (1965).

7 J. C. Stewart, I. Kuščer, and N. J. McCormick (to be published).

8 J. R. Mika, Nucl. Sci. Eng. 11, 415 (1961).

N. J. McCormick and I. Kusčer, J. Math. Phys. 7, 2036 (1966).
} 